\title{
New dominant spa type t2741 in livestock- associated MRSA (CC398-MRSA-V) in Finnish fattening pigs at slaughter
}

\author{
Annamari Heikinheimo', Sophia Johler², Laura Karvonen', Jérôme Julmi², Maria Fredriksson-Ahomaa'
} and Roger Stephan ${ }^{2 *}$

\begin{abstract}
Background: The emergence of livestock-associated MRSA has become a growing public health concern worldwide. Studies elucidating the population structure, as well as resistance phenotypes and virulence gene profiles of livestockassociated MRSA strains are needed to improve risk assessment and to develop effective control measures. The objective of this study therefore was to determine i) clonal complexes and spa types, as well as ii) resistance phenotypes and iii) virulence and resistance gene profiles of livestock-associated MRSA isolated from Finnish fattening pigs at slaughter.
\end{abstract}

Methods: Fifty MRSA isolates collected from Finnish fattening pigs at slaughter were characterized by spa typing and DNA microarray profiling. In addition, antimicrobial susceptibility testing was performed using the Kirby Bauer disk diffusion method.

Results: MRSA isolates were assigned to clonal complexes CC1 $(n=4)$ and CC398 $(n=46)$. One dominant spa type (t2741) was present in 33 out of 50 investigated isolates, originating from 15 out of 18 farms. The remaining isolates were assigned to spa types t034 $(n=7)$, t108 $(n=5)$, and t011 $(n=1)$. Although each herd exhibited isolates assigned to one clonal complex only, five herds harbored MRSA isolates of either two or three different spa types. All tested MRSA isolates were phenotypically resistant to penicillin, oxacillin, cefoxitin, and tetracycline. With the exception of the isolates assigned to t108, all isolates exhibited resistance to clindamycin. On the genomic level, all isolates exhibited mecA, blaZII/R, and tetK, and were assigned to SCCmec type V. Many isolates also harbored tetM (46/50 isolates), InuB (41/50 isolates), ermB (26/50 isolates), and one isolate was positive for aadD. DNA microarray profiling showed that all isolates of the dominant CC398/t2741 MRSA-V type belonged to agr type I, capsule type 5, and were negative for fnbB. Interestingly, one isolate of CC398/t2741 MRSA-V was agr negative and also lacked hld.

Conclusions: A new dominant LA-MRSA clone (CC398/t2741, SCCmec type V) was identified among fattening pigs in Finland. This is the first study identifying t2741 as a common spa type in LA-MRSA in pigs.

Keywords: Livestock-associated MRSA, Pig, Spa type 2741, fnbB, agr

\footnotetext{
* Correspondence: stephanr@fsafety.uzh.ch

${ }^{2}$ Institute for Food Safety and Hygiene, Vetsuisse Faculty University of Zurich,

Winterthurerstrasse 272, 8057 Zurich, Switzerland

Full list of author information is available at the end of the article
} 


\section{Background}

Methicillin-resistant Staphylococcus aureus (MRSA) is one of the leading causes of nosocomial infections and was first reported in pigs in 2005 [1]. The European Centre for Disease Prevention and Control reported pronounced inter-country variation (0.9-56 \%) with regard to the occurrence of MRSA infections in humans in Europe, with Finland exhibiting a very low percentage of invasive MRSA isolates ( $<5 \%)$ [2]. However, while the proportion of MRSA isolates in all invasive Finnish isolates tested declined from 2011 to 2013, this trend was reversed in 2014 [2].

In the last decade, livestock-associated MRSA (LAMRSA) have emerged worldwide. LA-MRSA are mostly associated with clonal complex CC398 and can be found at especially high prevalence rates in pigs [3-6]. In Finland, LA-MRSA emerged in 2007, but the proportion of CC398 MRSA in all MRSA isolates detected in humans was very low (0.09\% for 2007-2009) [7]. Data on the prevalence and population structure of Finnish LA-MRSA isolates from pigs is extremely limited. However, seven porcine CC398 isolates obtained in 2008 and 2009 were typed and were exclusively assigned to spa type t108 [7].

Epidemiological studies indicate that LA-MRSA also represents an increasing cause of infections in humans [8], LA-MRSA CC398 isolates are likely to have originated in humans as methicillin-susceptible Staphylococcus aureus. It has been suggested that while the jump from humans to livestock was followed by acquisition of tetracycline and methicillin resistance, it also led to decreased capacity for human colonization and transmission [9].

MRSA-ST398 isolates were reported to lack important virulence factors such as $p v l$, but were suggested to exhibit enhanced ability to acquire mobile genetic elements [10]. Consistent with this hypothesis, first cases of hospitalizations and death due to infections with MRSAST398 isolates exhibiting Panton-Valentine Leukocidin have recently been described $[11,12]$. Determining the population structure, as well as resistance phenotypes and virulence gene profiles of LA-MRSA isolates therefore plays a crucial role in risk assessment and provides data essential to the development of effective control measures.

This study aimed to determine i) clonal complexes and spa types, as well as ii) resistance phenotypes and iii) virulence and resistance gene profiles of LA-MRSA isolated from Finnish fattening pigs at slaughter.

\section{Methods}

\section{Sampling, bacterial isolation, and DNA extraction}

A total of 29 pig herds from 29 different pig farms from the most dense pig production area of Finland, Western Finland, were included in the study. The farms that the pigs originated from harbored large herds that surpassed average herd size for pig farms in Finland. Some farms obtained the pigs from one seller only, while others obtained pigs from several sellers. From June to July 2015, samples from the anterior nares of pigs at slaughter and from pig carcasses $(10 \mathrm{~cm} \times 10 \mathrm{~cm}$ surface sampling) were obtained using dry swabbing (Amies Transport Medium, Copan, Brescia, Italy). For each herd, 20 nasal swabs from pigs and 10 swabs from carcasses were taken. Five swabs each were subsequently pooled, resulting in four pooled pig nasal swab samples and two pooled carcass swab samples per herd. Samples were pre-enriched in Mueller-Hinton Broth with $6.5 \% \mathrm{NaCl}$, incubated at $37{ }^{\circ} \mathrm{C}$ for $16-24 \mathrm{~h}$. A total of $1 \mathrm{ml}$ was transferred to $9 \mathrm{ml}$ Tryptic soy broth (St. Louis, MO, USA) with cefoxitin $(3.5 \mathrm{mg} / \mathrm{L})$ and aztreonam $(75 \mathrm{mg} / \mathrm{L})$. After incubation at $37{ }^{\circ} \mathrm{C}$ for $16-20 \mathrm{~h}$, a total of $10 \mu \mathrm{l}$ was transferred on ChromAgar MRSA (Labema, Paris, France) and incubated at $37{ }^{\circ} \mathrm{C}$ for $24-48 \mathrm{~h}$. Suspect colonies (one colony from each sample) were confirmed with API Staph (Biomerieux, Paris, France). The antimicrobial susceptibility testing was done by disc diffusion method using cefoxitin discs (EUCAST ecological cut-off values) and PBP2' test (Oxoid, Hampshire, UK).

A total of 50 MRSA were isolated from nasal swabs of fattening pigs $(n=49)$ and a pig carcass $(n=1)$, originating from 18 different herds/farms. MRSA isolates were grown on $5 \%$ sheep blood agar at $37{ }^{\circ} \mathrm{C}$ overnight and chromosomal DNA was extracted using the Qiagen DNeasy Blood and Tissue Kit (Hilden, Germany) in accordance with the manufacturer's instructions.

\section{DNA microarray profiling}

All presumptive MRSA isolates were further characterized using the Staphytype genotyping 2.0 microarray (Alere, Jena, Germany), which determines the presence or absence of over 300 virulence and resistance genes and their allelic variants. The DNA microarray can also be used as a tool for species confirmation and allows for assignment of isolates to SCCmec types and clonal complexes [13]. The similarity of the resulting resistance and virulence gene profiles of the different MRSA isolates was visualized using SplitsTree4 (http://www.splitstree.org/) as previously described [14].

\section{spa typing}

All isolates were characterized with spa typing [15] and spa types were assigned using Bionumerics software version 7.5 (Applied Maths, Sint-Martens-Latem, Belgium).

\section{PCR screening for InuB}

Screening for the occurrence of $\ln u B$ was done by PCR according to Lozano et al. [16]. 


\section{Antimicrobial susceptibility testing}

Antimicrobial susceptibility testing was performed for eight antimicrobial agents belonging to seven antibiotic classes including anti-staphylococcal beta-lactams (penicillin, oxacillin, cefoxitin), aminoglycosides (gentamicin), macrolides (erythromycin), lincosamides (clindamycin), tetracyclines (tetracycline), fluoroquinolones (ciprofloxacin), and rifamycins (rifampicin) using the Kirby Bauer disk diffusion method. Results were interpreted according to the Clinical and Laboratory Standards Institute [17].

\section{Results}

An overview of the typing and characterization results is provided in Table 1. The 50 MRSA isolates were assigned to clonal complexes $\mathrm{CC} 1(n=4)$ and CC398 $(n=46)$, comprising isolates of highly similar resistance and virulence gene profiles (Fig. 1). We identified one dominant spa type (t2741) in 33 out of 50 investigated isolates, originating from 15 out of 18 farms. The remaining isolates were assigned to spa types t034 $(n=7), \mathrm{t} 108(n=5)$, and t011 $(n=1)$. Although each herd harbored only isolates assigned to one clonal complex, we detected either two or three different spa in five of the herds. Herd $\mathrm{H} 2$ was the only herd harboring MRSA of a clonal complex other than CC398, with all four MRSA isolates assigned to CC1 (spa types t127 and t1381).

All tested MRSA isolates were phenotypically resistant to penicillin, oxacillin, cefoxitin, and tetracycline. With the exception of the isolates assigned to $\mathrm{t} 108$, all isolates also exhibited resistance to clindamycin. On the genomic level, all isolates exhibited mecA, blaZ/I/R, and tet $K$, and were assigned to SCCmec type V (Table 1). Many isolates also harbored tetM (46/50 isolates), $\ln u B$ (41/50 isolates), ermB (26/50 isolates), and one isolate was positive for $a a d D$.

DNA microarray profiling showed that all isolates of the dominant CC398/t2741 MRSA-V type belonged to agr type I, capsule type 5 , and were negative for $f n b B$ encoding fibronectin-binding protein $\mathrm{B}$ (FnBPB). In general, most of the investigated strains lacked several important virulence genes. None of the investigated MRSA isolates carried genes encoding Panton Valentine Leukocidin $(p v l)$, toxic shock syndrome toxin (tst1), exfoliative toxins $(e t A / B / D)$, chemotaxis-inhibiting protein (chp), staphylococcal complement inhibitor $(s c n)$, staphylokinase (sak), epidermal cell differentiation inhibitors (edin $A / B / C$ ). In addition, with the exception of the $\mathrm{CC} 1$ strains exhibiting seh, no enterotoxins genes were detected.

Two major subclones were identified among the isolates of the dominant CC398/t2741 type: The first subclone $(n=19$, detected in 11 herds) exhibited phenotypic resistance to penicillin, oxacillin, cefoxitin, tetracycline, clindamycin, and erythromycin. In contrast, the second subclone ( $n=12$, detected in 4 herds) lacked erythromycin resistance and the ermB gene. Only one herd (H1) harbored both subclones. Interestingly, one isolate of CC398/t2741 MRSA-V exhibited genomic and phenotypic traits highly similar to the first subclone, was agr negative and also lacked hld. A comprehensive compilation of source data, as well as the characterization results obtained by DNA microarray profiling, PCR assays targeting spa and $\ln u B$, and

Table 1 Characteristics of 50 S. aureus isolates collected from fattening pigs at slaughter in Finland

\begin{tabular}{|c|c|c|c|c|c|c|c|c|c|c|}
\hline CC & spa typing & sccmec & Herd & Resistance phenotype ${ }^{a}$ & Resistance genes $^{\mathrm{b}}$ & $\ln u B$ & $\mathrm{se}^{\mathrm{c}}$ & $f n b B$ & agr & cap \\
\hline \multirow[t]{2}{*}{$\operatorname{CC1}(n=4)$} & $\mathrm{t} 127(n=1)$ & V & $\mathrm{H} 2$ & PEN, OXA, FOX, TET, CLI, ERY & mecA, blaZ/I/R, tetK, ermB, & - & seh & + & agrlll & cap8 \\
\hline & $\mathrm{t} 1381(n=3)$ & V & $\mathrm{H} 2$ & PEN, OXA, FOX, TET, CLI, ERY & mecA, blaZ/I/R, tetK, ermB & - & seh & + & agrlll & cap8 \\
\hline \multirow[t]{8}{*}{ CC398 $(n=46)$} & t011 $(n=1)$ & V & $\mathrm{H} 18$ & PEN, OXA, FOX, TET, CLI, ERY & mecA, blaZ/I/R, tetKMM, ermB & + & - & - & agrl & cap5 \\
\hline & t034 $(n=7)$ & V & $\mathrm{H} 1, \mathrm{H} 7, \mathrm{H} 12, \mathrm{H} 18$ & $\begin{array}{l}n=6: \text { PEN, OXA, FOX, } \\
\text { TET, CLI }\end{array}$ & mecA, blaZ/I/R, tetKM & + & - & + & agrl & cap5 \\
\hline & & & $\mathrm{H} 10$ & $\begin{array}{l}n=1: \text { PEN, OXA, FOX, TET, } \\
C L I, C I P\end{array}$ & mecA, blaZ/I/R, tetKMM, aadD & + & - & + & agrl & cap5 \\
\hline & $\mathrm{t} 108(n=5)$ & V & $\mathrm{H} 3, \mathrm{H} 16$ & PEN, OXA, FOX, TET & mecA, blaZ/I/R, tetKMM & - & - & + & agrl & cap5 \\
\hline & $\mathrm{t} 2741(n=33)$ & V & $\begin{array}{l}\mathrm{H} 1, \mathrm{H} 5, \mathrm{H6}, \mathrm{H} 7, \\
\mathrm{H} 8, \mathrm{H} 9, \mathrm{H} 11, \mathrm{H} 13 \\
\mathrm{H} 14, \mathrm{H} 17, \mathrm{H} 18\end{array}$ & $\begin{array}{l}n=19: \text { PEN, OXA, FOX, TET, } \\
\text { CLI, ERY }\end{array}$ & mecA, blaZII/R, tetKMM, ermB & + & - & - & agrl & cap5 \\
\hline & & & $\mathrm{H} 1, \mathrm{H} 4, \mathrm{H} 10, \mathrm{H} 15$ & $\begin{array}{l}n=12: \text { PEN, OXA, FOX, } \\
\text { TET, CLI }\end{array}$ & mecA, blaZ/I/R, tetKM & + & - & - & agrl & cap5 \\
\hline & & & $\mathrm{H} 9$ & $\begin{array}{l}n=1: \text { PEN, OXA, FOX, TET, } \\
C L I, E R Y, C I P\end{array}$ & mecA, blaZII/R, tetKMM, ermB & + & - & - & agrl & cap5 \\
\hline & & & $\mathrm{H} 17$ & $\begin{array}{l}n=1: \text { PEN, OXA, FOX, TET, } \\
\text { CLI, ERY }\end{array}$ & mecA, blaZII/R, tetKMM, ermB & + & - & - & - & cap5 \\
\hline
\end{tabular}

aPEN: penicillin, OXA: oxacillin, FOX: cefoxitin, TET: tetracycline, CLI: clindamycin, ERY: erythromycin; CIP: ciprofloxacin

${ }^{b}$ Selected resistance genes detected by DNA microarray (mecA, blaZ/I/R, ermB, tetK/M, aadD) or PCR (InuB)

cse: genes encoding staphylococcal enterotoxins 


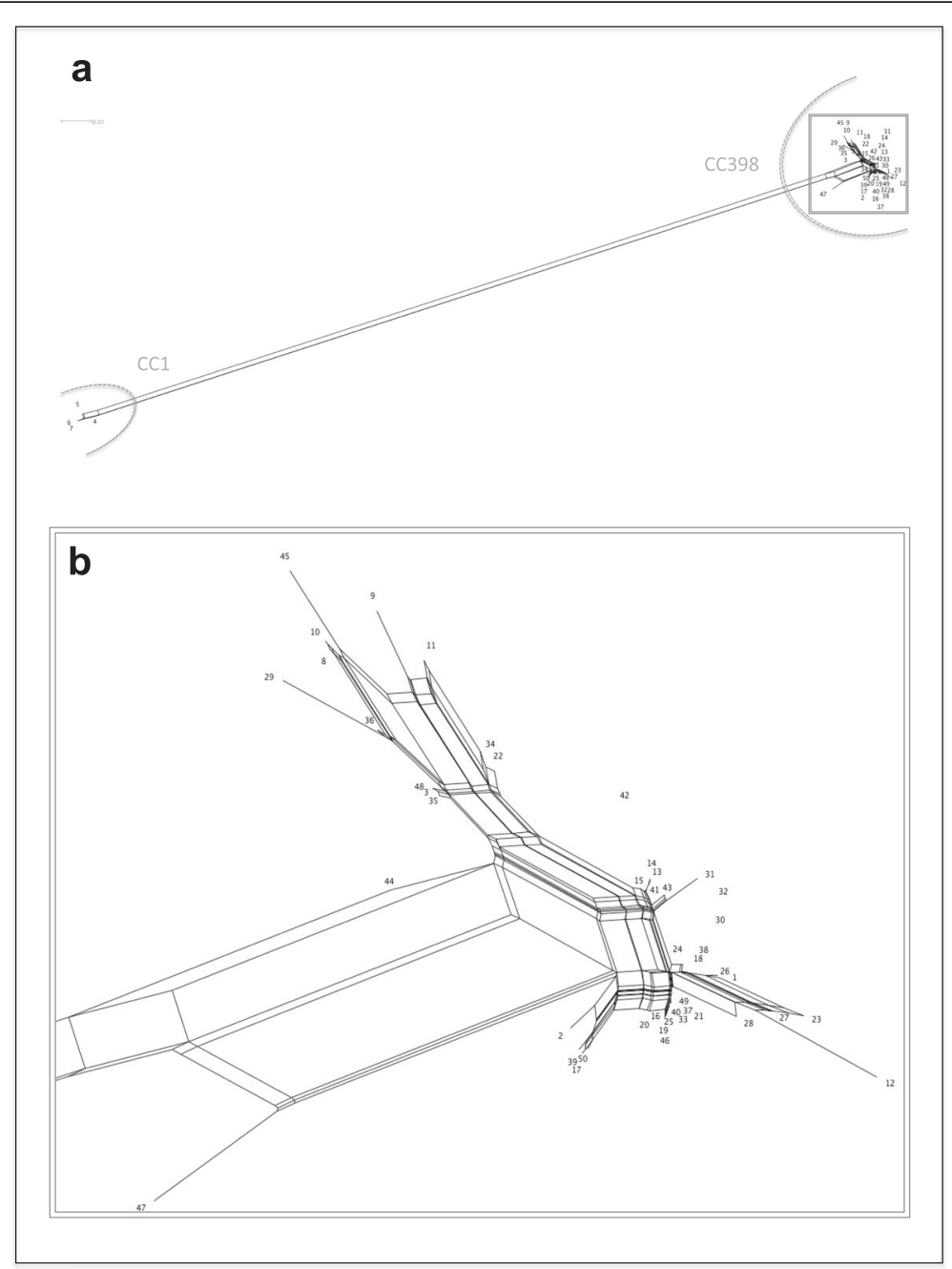

Fig. 1 SplitsTree visualizing the similarity of the DNA microarray profiles of the 50 MRSA isolates obtained from fattening pigs in Finland. a Overview over the full SplitsTree depicting all 50 isolates. b Detail zooming in on the region depicting the 46 highly similar CC398 isolates, while omitting the four CC1 isolates

the antimicrobial susceptibility testing is provided as a Additional files 1 and 2.

\section{Discussion}

Isolates of CC398/t2741 exhibiting SCCmec type V were dominant among the investigated MRSA isolates. To date, few individual CC398/t2741 MRSA-V isolates have been reported in association with human hosts $[8,18,19]$. The National Institute of Health and Welfare reported in its infection news that while $72 \%$ of the recent CC398associated infections in humans in Finland were caused by strains of spa type t034, $7 \%$ of the infections were associated with t2741 strains (https://www.thl.fi/en/web/thlfien). However, this is the first study identifying t2741 as a common spa type in LA-MRSA in pigs. In addition, no fnbB-negative or agr/hld-negative t2741 isolates have been previously reported.
Previous studies suggest that MRSA-CC398 vary only very little in core genome-encoded surface and secreted genes, with $f n b B$ being absent in only 1 out of 76 analyzed isolates [20]. FnBPB belongs to the microbial surface components recognizing adhesive matrix molecules (MSCRAMMs). It enables S. aureus to adhere to components of host cells and plays a role in invasion [21]. The combined function of FnBPA and FnBPB was shown to be essential for the induction of severe infection [22]. The accessory gene regulator $(a g r)$ represents a quorum sensing system mediating the staphylococcal virulon. While dysfunction of the agr system was suggested to promote proliferation and survival of $S$. aureus within the infected host, it may be counter-adaptive outside of infected host tissues and may decrease the chances of long-term survival [23].

The other spa types detected in this study (t011, t034, t108) are commonly found in LA-MRSA isolated from 
pigs in Europe [3, 19, 24-26]. Interestingly, t108 was shown to be transmissible between pigs and pig farmers, as well as between humans [1]. There are also indications that spa type t108 might have been the predominant spa type in LA-MRSA strains isolated from Finnish pigs, when CC398 first emerged in Finland [7].

Nineteen of the MRSA CC398 isolates (t034, t2741) in this study exhibited the unusual resistance phenotype $\mathrm{L}^{\mathrm{R}} / \mathrm{M}^{\mathrm{S}}$ (lincosamide resistance, macrolide susceptibility). These isolates were exclusively associated with spa types t034 (7/7 isolates) and t2741 (12/33 isolates). Recently, this unusual resistance phenotype has increasingly been observed among strains from animal origin [27]. In MRSA CC398 isolates lacking erm genes, this resistance phenotype has been suggested to be conferred by $\ln u A$ / $B / C / D / G$ [16]. This is consistent with the findings in this study, showing that the $L^{R} / M^{S}$ phenotype was associated with isolates lacking ermB, but harboring $\ln u B$.

The fact that only very few enterotoxin genes and no $p v l$, et $A / B / D$, and tst-1 genes were detected in this study is consistent with previous studies indicating that $S$. aureus of ST398 lack certain virulence factors, which may account for these isolates being only rarely associated with invasive disease in humans (Schijffelen et al., 2010). However, MRSA ST398 isolates were suggested to exhibit an enhanced ability to acquire mobile genetic elements and may therefore rapidly acquire virulence determinants increasing the virulence of these strains in the human host [10]. As first cases of severe illness and death due to infections with MRSA-ST398 isolates exhibiting Panton-Valentine Leukocidin have been described [11, 12], the occurrence of MRSA ST398 isolates in livestock and human hosts and associated virulence and resistance gene patterns needs to be closely monitored.

\section{Conclusions}

A new dominant LA-MRSA clone (CC398/t2741, SCCmec type V) was identified among fattening pigs in Finland. This is the first study identifying t2741 as a common spa type in LA-MRSA in pigs. As strains of ST398 were reported to easily acquire virulence determinants, monitoring of virulence and resistance profiles of LA-MRSA in livestock and human carriers in close contact to livestock production is crucial to recognize emerging hypervirulent clones and to develop comprehensive and efficient control strategies.

\section{Statement on ethics}

There is no consideration about the sampling of carcasses because animal rights do not apply to slaughtered farm animals. Nasal swabs were taken after stunning and therefore comply with national guidelines.

\section{Additional files}

Additional file 1: Overview on phenotypic and genotypic characteristics of each strain. (XLSX $31 \mathrm{~kb}$ )

Additional file 2: Complete microarray data for each strain. (XLSX 86 kb)

\section{Competing interests}

The authors declare that they have no competing interests.

\section{Authors' contributions}

$\mathrm{AH}$ and MF designed the study. Laboratory work was carried out by LK, $A H$, $J J$ and RS and results were interpreted by SJ, RS, and AH. SJ and RS drafted the manuscript. All authors read and approved the final manuscript.

\section{Acknowledgments}

We thank the staff of the slaughterhouses for facilitating access to their operations. Moreover, we would like to thank Jari Jalava from The National Institute for Health and Welfare (Finland) for providing control strains for this study.

\section{Author details}

${ }^{1}$ Department of Food Hygiene and Environmental Health, Faculty of Veterinary Medicine, University of Helsinki, Agnes Sjöberginkatu 2, P.O. Box 66, Fl 00014 Helsinki, Finland. ${ }^{2}$ Institute for Food Safety and Hygiene, Vetsuisse Faculty University of Zurich, Winterthurerstrasse 272, 8057 Zurich, Switzerland

Received: 22 January 2016 Accepted: 23 February 2016 Published online: 02 March 2016

\section{References}

1. Voss A, Loeffen F, Bakker J, Klaassen C, Wulf M. Methicillin-resistant Staphylococcus aureus in pig farming. Emerg Infect Dis. 2005;11:1965-6.

2. European Centre for Disease Prevention and Control. Antimicrobial resistance surveillance in Europe 2014. Annual report of the European Antimicrobial Resistance Surveillance Network (EARS-Net). Stockholm: ECDC; 2015

3. de Neeling AJ, van den Broek MJM, Spalburg EC, van Santen-Verheuvel MG, Dam-Deisz WDC, Boshuizen HC, van de Giessen AW, van Duijkeren E, Huijsdens XW. High prevalence of methicillin resistant Staphylococcus aureus in pigs. Vet Microbiol. 2007;122:366-72.

4. Khanna T, Friendship R, Dewey C, Weese JS. Methicillin resistant Staphylococcus aureus colonization in pigs and pig farmers. Vet Microbiol. 2008;128:298-303.

5. Smith TC, Male MJ, Harper AL, Kroeger JS, Tinkler GP, Moritz ED, Capuano AW, Herwaldt LA, Diekema DJ. Methicillin-resistant Staphylococcus aureus (MRSA) strain ST398 is present in midwestern U.S. swine and swine workers. PLoS ONE. 2009;4:e4258.

6. Alt K, Fetsch A, Schroeter A, Guerra B, Hammerl J, Hertwig S, Senkov N, Geinets A, Mueller-Graf C, Braeunig J, Kaesbohrer A, Appel B, Hensel A, Tenhagen BA. Factors associated with the occurrence of MRSA CC398 in herds of fattening pigs in Germany. BMC Vet Res. 2011;7:69.

7. Salmenlinna S, Lyytikäinen O, Vainio A, Myllyniemi A-L, Raulo S, Kanerva M, Rantala M, Thomson K, Seppänen J, Vuopio J. Human cases of methicillinresistant Staphylococcus aureus CC398 infection, Finland. Emerg Infect Dis. 2010;16:1626-9.

8. Köck R, Schaumburg F, Mellmann A, Köksal M, Jurke A, Becker K, Friedrich AW. Livestock-associated methicillin-resistant Staphylococcus aureus (MRSA) as causes of human infection and colonization in Germany. PLoS ONE. 2013;8, e55040.

9. Price LB, Stegger M, Hasman H, Aziz M, Larsen J, Andersen S, Pearson T. Adaptation and emergence of Staphylococcus aureus CC398: Host adaptation and emergence of methicillin resistance in livestock. MBio. 2012;3:1-6.

10. Schijffelen MJ, Boel CHE, van Strijp JAG, Fluit AC. Whole genome analysis of a livestock-associated methicillin-resistant Staphylococcus aureus ST398 isolate from a case of human endocarditis. BMC Genomics. 2010;11:376.

11. Welinder-Olsson C, Floren-Johansson K, Larsson L, Öberg S, Karlsson L, Ahren C. Infection with Panton-Valentine leukocidin-positive methicillinresistant Staphylococcus aureus t034. Emerg Infect Dis. 2008;14:1271-2. 
12. Koyama H, Sanui M, Saga T, Harada S, Ishii Y, Tateda K, Lefor AK. A fatal infection caused by sequence type 398 methicillin-resistant Staphylococcus aureus carrying the Panton-Valentine leukocidin gene: a case report in Japan. J Infect Chemother. 2015;21:541-3.

13. Monecke $S$, Slickers $P$, Ehricht R. Assignment of Staphylococcus aureus isolates to clonal complexes based on microarray analysis and pattern recognition. FEMS Immunol Med Microbiol. 2008;53:237-51.

14. Wattinger $L$, Stephan R, Layer F, Johler S. Comparison of Staphylococcus aureus isolates associated with food intoxication with isolates from human nasal carriers and human infections. Eur J Clin Microbiol Infect Dis. 2012;31:455-64.

15. Shopsin B, Eaton C, Wasserman GA, Mathema B, Adhikari RP, Agolory S, Altman DR, Holzman RS, Kreiswirth BN, Novick RP. Mutations in agr do not persist in natural populations of methicillin-resistant Staphylococcus aureus. J Infect Dis. 2010;202:1593-9.

16. Lozano C, Aspiroz C, Sáenz Y, Ruiz-García M, Royo-García G, Gómez-Sanz E, Ruiz-larrea F, Zarazaga M, Torres C. Genetic environment and location of the Inu(A) and Inu(B) genes in methicillin-resistant Staphylococcus aureus and other staphylococci of animal and human origin. J Antimicrob Chemother. 2012;67:2804-8.

17. Clinical and Laboratory Standards Institute (CLSI). Performance Standards for Antimicrobial Disk and Dilution Susceptibility Tests for Bacteria Isolated From Animals; Approved Standard-Third Edition. CLSI document M31-A3 (ISBN 1-56238-659-X). Clinical and Laboratory Standards Institute, 940 West Valley Road, Suite 1400, Wayne, Pennsylvania 19087-1898 USA, 2008.

18. Reischl U, Frick J, Hoermansdorfer S, Melzl H, Bollwein M, Linde HJ, Becker K, Köck R, Tuschak C, Busch U, Sing A. Single-nucleotide polymorphism in the SCCmec-orfX junction distinguishes between livestock-associated MRSA 398 and human epidemic MRSA strains. Medicine. 2009;14:1-8.

19. van den Broek IVF, van Cleef BAGL, Haenen A, Broens EM, van der Wolf PJ, van den Broek MJM, Huijsdens XW, Kluytmans JAJW, van de Giessen AW, Tiemersma EW. Methicillin-resistant Staphylococcus aureus in people living and working in pig farms. Epidemiol Infect. 2009;137:700-8.

20. McCarthy AJ, Witney A, Gould K, Moodley A, Guardabassi L, Voss A, Denis O, Broens EM, Hinds J, Lindsay J. The distribution of mobile genetic elements (MGEs) in MRSA CC398 is associated with both host and country. Genome Biol Evol. 2011:3:1164-74.

21. Foster TJ, Geoghegan JA, Ganesh VK, Höök M. Adhesion, invasion and evasion: the many functions of the surface proteins of Staphylococcus aureus. Nat Rev Microbiol. 2013;12:49-62.

22. Shinji H, Yosizawa Y, Tajima A, Iwase T, Sugimoto S, Seki K, Mizunoe Y. Role of fibronectin-binding proteins $A$ and $B$ in in vitro cellular infections and in vivo septic infections by Staphylococcus aureus. Infect Immun. 2011;79:2215-23.

23. Shopsin B, Gomez M, Montgomery SO, Smith DH, Waddington M, Dodge DE, Bost DA, Riehman M, Naidich S, Kreiswirth BN. Evaluation of protein A gene polymorphic region DNA sequencing for typing of Staphylococcus aureus strains. J Clin Microbiol. 1999;37:3556-63.

24. Huber H, Koller S, Giezendanner N, Stephan R, Zweifel C. Prevalence and characteristics of meticillin-resistant Staphylococcus aureus in humans in contact with farm animals, in livestock, and in food of animal origin, Switzerland, 2009. Euro Surveill. 2010;15:1-4.

25. Broens EM, Graat EM, Van der Wolf PJ, Van de Giessen AW, Van Duijkeren E, Wagenaar JA, Van Nes A, Mevius DJ, de Jong MCM. MRSA CC398 in the pig production chain. Prev Vet Med. 2011;98:182-9.

26. Anonymous. EU Summary Report on antimicrobial resistance in zoonotic and indicator bacteria from humans, animals and food in 2013. EFSA J. 2015;13:4036.

27. Hauschild T, Feßler AT, Kadlec K, Billerbeck C, Schwarz S. Detection of the novel vga(E) gene in methicillin-resistant Staphylococcus aureus CC398 isolates from cattle and poultry. J Antimicrob Chemother. 2012;67:503-4.

\section{Submit your next manuscript to BioMed Central and we will help you at every step:}

- We accept pre-submission inquiries

- Our selector tool helps you to find the most relevant journal

- We provide round the clock customer support

- Convenient online submission

- Thorough peer review

- Inclusion in PubMed and all major indexing services

- Maximum visibility for your research

Submit your manuscript at www.biomedcentral.com/submit
Biomed Central 